\title{
kth Power of a Partial Sum
}

\section{Angel Plaza}

To cite this article: Angel Plaza (2019) kth Power of a Partial Sum, The American Mathematical Monthly, 126:5, 467-467, DOI: 10.1080/00029890.2019.1577087

To link to this article: https://doi.org/10.1080/00029890.2019.1577087

$$
\text { 曲 Published online: } 10 \text { May } 2019 .
$$

Submit your article to this journal $\widetilde{ }$

Џ Article views: 302

Q View related articles $₫$

View Crossmark data $\nearrow$ 


\section{kth Power of a Partial Sum}

Recently the following result appeared [1, Theorem 2.1].

Theorem 1. For any finite sequence of positive numbers $\left(a_{j}\right)_{j=1}^{n}$ whose partial sums are $\left(S_{j}\right)_{j=1}^{n}$ we have $\sum_{j=1}^{n}\left(a_{j}^{2}+2 a_{j} S_{j-1}\right)=S_{n}^{2}$.

Here we prove the following extension of the previous result.

Theorem 2. For any finite sequence of positive numbers $\left(a_{j}\right)_{j=1}^{n}$ whose partial sums are $\left(S_{j}\right)_{j=1}^{n}$ and for all integers $k \geq 1$ we have

$$
\sum_{j=1}^{n} \sum_{m=1}^{k}\left(\begin{array}{l}
k \\
m
\end{array}\right) S_{j-1}^{k-m} a_{j}^{m}=S_{n}^{k}
$$

Proof. The proof follows by telescoping. Since $S_{j}=S_{j-1}+a_{j}$, we have

$$
S_{j}^{k}-S_{j-1}^{k}=\left(S_{j-1}+a_{j}\right)^{k}-S_{j-1}^{k}=\sum_{m=1}^{k}\left(\begin{array}{c}
k \\
m
\end{array}\right) S_{j-1}^{k-m} a_{j}^{m} .
$$

Since $S_{0}=0$, we have $\sum_{j=1}^{n} \sum_{m=1}^{k}\left(\begin{array}{c}k \\ m\end{array}\right) S_{j-1}^{k-m} a_{j}^{m}=\sum_{j=1}^{n}\left(S_{j}^{k}-S_{j-1}^{k}\right)=S_{n}^{k}$.

Corollary. For $k=3$ we have $\sum_{j=1}^{n}\left(a_{j}^{3}+3 a_{j}^{2} S_{j-1}+3 a_{j} S_{j-1}^{2}\right)=S_{n}^{3}$.

Example. Let $a_{j}=F_{2 j-1}$. It is well known that $S_{n}=\sum_{j=1}^{n} F_{2 j-1}=F_{2 n}$. It follows that $a_{j}^{3}+3 a_{j}^{2} S_{j-1}+3 a_{j} S_{j-1}^{2}=F_{2 j-1}^{3}+3 F_{2 j-1} F_{2 j-2} F_{2 j}$, which implies

$$
\sum_{j=1}^{n}\left(F_{2 j-1}^{3}+3 F_{2 j-1} F_{2 j-2} F_{2 j}\right)=F_{2 n}^{3} \text {. }
$$

Many other identities may be found and proved using Theorem 2.

\section{REFERENCE}

[1] Treeby, D. (2016). Further physical derivations of Fibonacci summations. Fibonacci Quart. 54(4): $327-334$.

— Submitted by Angel Plaza, University of Las Palmas de Gran Canaria, Spain

doi.org/10.1080/00029890.2019.1577087

MSC: Primary 11B39, Secondary 11B37; 11B65 